\title{
Deceptive Actions and Demonstrations of Intentions for Robot Collision Avoidance
}

\author{
Vladimir Popov ${ }^{1, a)}$ and Anna Gorbenko ${ }^{1, b)}$ \\ ${ }^{1}$ Department of Intelligent Systems and Robotics, Institute of Natural Sciences and Mathematics, Ural Federal \\ University, 620083 Ekaterinburg, Lenin st., 51, Russia. \\ a)Corresponding author: popovvvv@gmail.com \\ b) gorbenko.aa@gmail.com
}

\begin{abstract}
Efficiency and effectiveness of many robotics tasks depend crucially on the quality of the solution of the problem of collision avoidance. In many cases, traditional path planning and obstacle avoiding approaches and algorithms can not guarantee a sufficiently high quality of the solution of the problem of collision avoidance for multi-robot and human-robot interactions. In this paper, we consider demonstrations of true intentions and demonstrations of deceptions for the problem of collision avoidance.
\end{abstract}

\section{INTRODUCTION}

Various problems of collision avoidance have received considerable attention (see e.g. [1, 2]). In particular, when robots and humans share the same environment, we need to solve the problem of human-robot collision avoidance to guarantee safe interactions between robots and humans. Also, collision between autonomous robots is one of the main problems in multi-robot task cooperation [3]. In general, collision avoidance is the task of steering free of collisions with static and dynamic obstacles, while following some global plan. It should be noted that static and dynamic obstacles require the use of significantly different methods and approaches. Moreover, the methods for solving the problem of collision avoidance depend essentially on the type of dynamic obstacles. Therefore, it is natural to consider the problem of collision avoidance separately for different types of dynamic obstacles. In this paper, we consider the multi-robot problem of collision avoidance. Also, we pay some attention to the human-robot problem of collision avoidance.

Different problems of multi-robot task cooperation are extensively studied in contemporary robotics (see e.g. $[4,5,6])$. There is a number of examples of deployments of an autonomous robot in an unscripted human environment (see e.g. [7, 8, 9]). Human-robot collaborations have received considerable attention (see e.g. [10, 11]). Nevertheless, problems of multi-robot and human-robot collision avoidance are still far from final solutions (see e.g. [12]). It should be noted that classical path planning and obstacle avoiding schemes may be too slow to provide sufficiently safe and efficient navigation (see e.g. [13]). Therefore, in general, we need to use some special schemes to guarantee safe collision avoidance. Moreover, in some cases, we need some additional scheme to ensure that the robot can resume its path after collision avoidance to still reach its goals effectively and efficiently [3].

Usually, humans can communicate via multiple channels like gaze, speech, gestures, movement and posture. Understanding and participating in such communication enables humans to predict an action rather than react to it. Moreover, humans can use additional channels to demonstrate their intentions. Understanding of intentions makes it much easier to solve many planning tasks. Usually, robots have significantly lower capabilities for communication. The use of additional channels in many cases is unacceptable for robots. Therefore, it is important to consider the ability of robots to demonstrate their intentions directly by their motion. Accordingly, robots should have some capabilities to extract the intentions of other robots directly from the motion. In this paper, we consider the problem of collision avoidance and the possibility of generating and using demonstrations of intentions to manipulate another robot or pedestrian. It is assumed that robots can use not only demonstrations of true intentions but also demonstrations of false intentions or deceptions. There is a number of robotic studies of the problem of deception (see e.g.

International Conference of Numerical Analysis and Applied Mathematics ICNAAM 2019

AIP Conf. Proc. 2293, 420032-1-420032-4; https://doi.org/10.1063/5.0027660

Published by AIP Publishing. 978-0-7354-4025-8/\$30.00 
$[14,15,16])$. In particular, robots can use deceptions for the problem of collision avoidance [17]. The main purpose of this paper is to compare the effectiveness of demonstrations of true intentions and demonstrations of deceptions.

\section{GENERATION OF MOTION AND TEST SCENARIOS}

We consider indoor environments that are systems of rooms and corridors. It is assumed that the environment contains only static obstacles. Robots should avoid these obstacles and collisions with targets and with each other. We are considering two mobile robots. The task of each robot is to follow the moving target. Each robot has its own target. So, we assume that successful demonstration of intentions itself is not a goal for the robot. It is assumed that a demonstration of intentions is used to improve the quality of the solution of the main task of the robot and the quality of the collision avoidance. It should be noted that we not consider path length as an objective. For each robot, the main objective is the time to solve the main task. It is assumed that in close proximity to each other robots should use special avoidance algorithms. The use of such algorithms leads to a significant lengthening of the trajectory of motion and as a consequence to a significant loss of time.

Robots use the same neural network path planning algorithm [18]. We use an adaptation of automatic generation of safety-critical test scenarios for collision avoidance of road vehicles [19].

In each scenario, it is assumed that the targets move relatively slowly along relatively simple paths. However, the presence of static obstacles provides for robots a good visibility only of their targets. Such conditions make it possible for robots to have a high predictability of the motion of their targets. However, robots do not have enough information to predict the motion of targets of other robots. Accordingly, robots cannot use the motion of targets of other robots to predict the trajectories of motion of other robots. Therefore, to predict the motion of other robots, the robots should be based only on the motion of these robots.

The robots are assumed in the same constant velocity. Therefore, to demonstrate the intentions, robots can only use changes in direction.

In general, we assume that robots solve their main tasks many times. Accordingly, robots can use previous experience. It is assumed that the motion of the robot can be divided into three phases, the robot motion phase before the collision avoidance, motion phase during the collision avoidance, motion phase after the collision avoidance. Besides main neural network path planning algorithm [18], the robots use two additional recurrent neural networks. The first network determines the appropriateness and the type of demonstration. The second neural network is used directly to generate a demonstration. To generate demonstrations, we use approaches that are close to the ideas from the paper [14]. To evaluate the performance of these neural networks, each scenario is performed three times, without demonstration, with demonstration of true intentions, with deceptive demonstration. The robots use recurrent neural networks for predicting the motion of another robot. During experiments, it is assumed that the neural network that determines the appropriateness and the type of demonstration is untrained. All other neural networks are pre-trained. To train the neural networks, we use computer simulations. For robotic experiments and for the generation of motion patterns for computer simulations, we use two types of robots, Neato Robotics Neato XV-11 with camera and Aldebaran Robotics robot Nao V4 H25. The robot Nao was used to simulate the motion of a pedestrian. In particular, experiment with Nao was performed under conditions that are similar to the conditions that are presented in the paper [20].

\section{EXPERIMENTAL RESULTS}

In our experiments, we consider two different neural networks as a network that determines the appropriateness and the type of demonstration. The neural network $N_{1}$ generates a number $X, X \in\{1,2,3\}$, such that $X=1$ means no need for a demonstration, $X=2$ means a demonstration of true intentions, $X=3$ means a demonstration of deception. The neural network $N_{2}$ generates a vector $(Y, Z),(Y, Z) \in\{(0,0),(0,1),(1,0),(1,1)\}$, such that $Y=0$ means no need for a demonstration, $Y=1$ means the need for a demonstration, $Z=0$ means a demonstration of true intentions, $Z=1$ means a demonstration of deception. Since each scenario is performed three times, we can evaluate the correctness of the predictions of neural networks $N_{1}$ and $N_{2}$. We use the backpropagation method to train these neural networks.

For each iteration of the backpropagation method and for verification, we consider 400 scenarios such that for 200 there is no need for a demonstration, for 100 it is necessary to demonstrate true intentions, for 100 it is necessary to demonstrate deception. In our first experiment, we assume that neural networks do not have information about the actions of another robot during previous interactions. For the verification after $n$ iterations, $T(U, n)$ denotes the number of correctly predicted values of $U$. Results of computer simulations are given in Table 1. 
TABLE 1. Two mobile robots such that neural networks do not have information about previous interactions.

\begin{tabular}{lccccc}
\hline$n$ & 50 & 100 & 150 & 200 & 400 \\
\hline$T(X, n), X=1$ & 118 & 159 & 178 & 193 & 196 \\
$T(X, n), X=2$ & 57 & 64 & 73 & 76 & 78 \\
$T(X, n), X=3$ & 81 & 88 & 95 & 97 & 96 \\
$T(Y, n), Y=0$ & 156 & 164 & 167 & 169 & 171 \\
$T(Z, n), Z=0$ & 53 & 59 & 62 & 64 & 61 \\
$T(Z, n), Z=1$ & 71 & 84 & 96 & 98 & 94 \\
\hline
\end{tabular}

It is clear that neural networks can emulate each other. Nevertheless, their training demonstrates significantly different results. In particular, the neural network $N_{2}$ for $Y=0$ trains significantly faster than the neural network $N_{1}$ for $X=1$. It should be noted that in both cases we have obtained significantly better results for deceptions than for demonstrations of true intentions.

When neural networks use information about previous interactions, it is difficult to estimate the need for demonstrations. Therefore, in this case, we cannot perform a completely analogous experiment. However, we can compare the average time efficiency. In our second experiment, we consider neural networks after 200 iterations for $m$ previous interactions. For each value of $m, T$ denotes the average time without demonstrations, $T_{f}$ denotes the average time with deceptions, $T_{t}$ denotes the average time with demonstrations of true intentions. It is assumed that the demonstrations are used by only one robot. For each value of $m$, we consider 400 scenarios for computer simulations and 20 for robotic experiments. Results of computer simulations and robotic experiments are given in Tables 2 and 3, respectively.

TABLE 2. Two mobile robots such that neural networks use information about previous interactions.

\begin{tabular}{lccccccc}
\hline$m$ & 0 & 5 & 10 & 15 & 20 & 25 & 30 \\
\hline$T_{f} / T$ & 0.272201 & 0.931272 & 1.028992 & 1.448235 & 1.833528 & 7.225248 & 7.376164 \\
$T_{t} / T$ & 0.891214 & 0.676638 & 0.624112 & 0.592216 & 0.562224 & 0.511243 & 0.455328 \\
\hline
\end{tabular}

TABLE 3. Neato with neural network that uses information about previous interactions and Nao.

\begin{tabular}{lccccccc}
\hline$m$ & 0 & 5 & 10 & 15 & 20 & 25 & 30 \\
\hline$T_{f} / T$ & 0.344742 & 0.321956 & 0.299428 & 0.276233 & 0.252218 & 0.248812 & 0.248106 \\
$T_{t} / T$ & 0.933342 & 0.926784 & 0.926324 & 0.902162 & 0.893881 & 0.845215 & 0.772331 \\
\hline
\end{tabular}

Despite the relatively low capabilities of our neural networks to correctly estimate the need for demonstrations of true intentions (see Table 1), the use of such demonstrations allows to achieve a sufficiently high result. Also it should be noted that the use of information about previous interactions results in a significant decrease in efficiency of deceptions for two mobile robots. However, for Nao that simulates the motion of a pedestrian, we do not observe a similar trend. The principal difference in the actions of Neato and Nao is that Neato takes into account the actions of another robot. At the same time, Nao only simulates the actions of a pedestrian.

\section{CONCLUSION}

The results of our experiments have shown that using deceptions is much easier than demonstrations of true intentions. Moreover, for human-robot collision avoidance, the use of deceptions allows to achieve a significantly better result. Nevertheless, the use of deceptions may be ineffective for long-term interaction of robots. 


\section{ACKNOWLEDGMENTS}

This work is partially supported by the Ministry of Education and Science of the Russian Federation project "Combinatorial models in computer science and their applications". The work was supported by Act 211 Government of the Russian Federation, contract N 02.A03.21.0006.

\section{REFERENCES}

[1] M. M. Almasri, A. M. Alajlan, and K. M. Elleithy, IEEE Sensors J. 16, 5021-5028 (2016).

[2] L. Zeng and G. M. Bone, Int. J. Adv. Robot. Syst. 10, 1-14 (2013).

[3] F. Liu and A. Narayanan, LNCS 8291, 181-196 (2013).

[4] M. Agarwal, N. Agrawal, S. Sharma, L. Vig, and N. Kumar, Expert Syst. Appl. 42, 7797-7811 (2015).

[5] Y. Li, L. Chen, K. P. Tee, and L. Q, Neurocomputing 170, 168-175 (2015).

[6] H. Oh, A. R. Shirazi, C. Sun, and Y. Jin, Robot. Auton. Syst. 91, 83-100 (2017).

[7] W. Burgard, A. B. Cremers, D. Fox, D. Hahnel, G. Lakemeyer, D. Schulz, W. Steiner, and S. Thrun, Artif. Intell. 114, 3-55 (1999).

[8] S. Thrun, M. Bennewitz, W. Burgard, A. B. Cremers, F. Dellaert, D. Fox, D. Hahnel, C. Rosenberg, N. Roy, J. Schulte, and D. Schulz, "Minerva: a second-generation museum tour-guide robot," in Proceedings of the 1999 IEEE International Conference on Robotics and Automation, Vol. 3 (IEEE Press, Piscataway, 1999), pp. 1999-2005.

[9] I. R. Nourbakhsh, C. Kunz, and T. Willeke, "The mobot museum robot installations: a five year experiment," in IEEE/RJS International Conference on Intelligent Robots and Systems (IEEE Press, Piscataway, 2003), pp. 3636-3641.

[10] B. Lacevic and P. Rocco, "Kinetostatic danger field - a novel safety assessment for human-robot interaction," in IEEE/RSJ International Conference on Intelligent Robots and Systems (IEEE Press, Piscataway, 2010), pp. 2169-2174.

[11] M. P. Polverini, A. M. Zanchettin, and P. Rocco, Robot. Comput. Integr. Manuf. 46, 25-37 (2017).

[12] P. Trautman, J. Ma, R. M. Murray, and A. Krause, Int. J. Rob. Res. 34, 335-356 (2015).

[13] S. Lo, C. Cheng, and H. Huang, J. Intell. Robot. Syst. 82, 3-19 (2016).

[14] A. Dragan, R. Holladay, and S. Srinivasa, Auton. Robots 39, 331-345 (2015).

[15] S. Gerwehr and R. W. Glenn, The art of darkness: deception and urban operations (Rand Corporation, Santa Monica, 2000).

[16] A. R. Wagner and R. C. Arkin, Int. J. of Soc. Robotics 3, 5-26 (2011).

[17] A. Gorbenko and V. Popov, "Deceptive actions and robot collision avoidance," in Biologically Inspired Cognitive Architectures 2019, Advances in Intelligent Systems and Computing 948, edited by A. V. Samsonovich (Springer, Cham, Switzerland, 2020), pp. 105-109.

[18] S. X. Yang and M. Meng, Neural Netw. 138, 143-148 (2000).

[19] M. Althoff and S. Lutz, "Automatic generation of safety-critical test scenarios for collision avoidance of road vehicles," in 2018 IEEE Intelligent Vehicles Symposium (IV) (IEEE Press, Piscataway, 2018), pp. 1-8.

[20] C. Vassallo, A. Olivier, P. Soueres, A. Cretual, O. Stasse, and J. Pettre, Gait \& Posture 51, 97-103 (2017). 